\title{
Narrowband UV-B phototherapy in childhood atopic dermatitis: efficacy and safety*
}

\author{
Surabhi Dayal ${ }^{1}$ \\ Priyadarshini Sahu ${ }^{1}$
}

\author{
Kalpana Pathak ${ }^{2}$ \\ Vijay Kumar Jain ${ }^{1}$
}

DOI: http:/ / dx.doi.org/10.1590/abd1806-4841.20175958

\begin{abstract}
BACKGROUND: Narrow-band UVB is the most innovative steroid sparing treatment in atopic dermatitis. There are studies showing efficacy of Narrow-band UVB in childhood atopic dermatitis, but there is lack of clinical trials in the literature determining the length of remission. Therefore, we sought to highlight its efficacy, safety and its post-treatment efficacy in childhood atopic dermatitis.

ОвјестіVE: To assess the clinical efficacy, safety of Narrow-band UVB in the treatment of paediatric atopic dermatitis and length of remission during 2 years of post-treatment follow-up.

METHODs: Thirty children (4-14 years) having moderate to severe AD (SCORAD index $>25)$ were enrolled for 12 weeks. Narrow-band UVB phototherapy was administered twice a week on non-consecutive days for three months. SCORAD index was calculated by the same dermatologist at baseline, 6th, 12th, 18th and 24th treatment session. Secondary outcomes were measured using visual analog scale for pruritus and sleep loss. Patients were also followed-up for 2 years to know the length of remission after end of therapy.

RESULTS: There was a significant reduction in SCORAD index at 6th, 12th, 18th and 24th treatment session in comparison to baseline. This improvement in SCORAD was also maintained during the 2 years of post-treatment follow-up period. Consequently, pruritus and sleep loss improved significantly from baseline to end of therapy and even during the 1st and 2nd year of follow-up.

STUDY LIMITATION: Open-label trial without control group.

CONCLUSIONS: Narrow-band UVB is an efficacious and safe modality of treatment in childhood atopic dermatitis with good therapeutic index and minimal side effects.
\end{abstract}

Keywords: Dermatitis, atopic; Phototherapy; Pruritus

\section{INTRODUCTION}

Atopic dermatitis (AD) is a common and distressing condition characterized by itchy, chronically relapsing inflammatory skin lesions associated with personal or family history of allergy. It is a major health problem worldwide with a prevalence of $15-20 \%$ in children and an increase by 2 - to 3 -fold in its incidence during the past few decades in industrialized countries has been observed. ${ }^{1}$ In chronic childhood $\mathrm{AD}$, emollients and topical steroids have been considered the mainstay of therapy. The long term adverse effects of topical steroids led to the development of nonsteroidal topical alternatives such as calcineurin inhibitors like tacrolimus and pimecrolimus. However, children with moderate to severe AD often fail to respond to topical therapies alone. Thus, a safe and effective treatment option is needed for moderate to severe childhood AD.
Phototherapy, particularly Narrow-band UVB (NBUVB) is considered as second line therapy for AD. ${ }^{2}$ A randomized controlled trial in adult eczema has shown NBUVB to be superior to other phototherapy modalities. ${ }^{3}$ As it has a good safety profile in children, it seems to be a good treatment option in childhood AD.

However, on reviewing the literature thoroughly we noticed that there are very limited studies available which have evaluated the efficacy of NBUVB phototherapy in the management of childhood AD. ${ }^{4-7}$ Previous studies by Collins et al. ${ }^{4}$, Clayton et al. ${ }^{5}$ and Darné $e t$ al . have emphasized and refurbished the therapeutic efficacy of NBUVB in childhood AD. There is only one study in Asian patients by Mok et al. ${ }^{7}$ who have also highlighted the usefulness of NBUVB in moderate to severe childhood AD; however, they

\section{Received on 22.04.2016.}

Approved by the Advisory Board and accepted for publication on 09.08.2016.

* Work performed at the Department of Dermatology, Venereology and Leprology, Pt B D Sharma Post Graduate Institute of Medical Sciences, Rohtak Haryana, India.

Financial support: None.

Conflict of interest: None.

\footnotetext{
Department of Dermatology, Venereology and Leprology, Pt. B. D. Sharma, Post Graduate Institute of Medical Sciences, Rohtak - Haryana, India. Skin Clinic, Ghaziabad, India
} 
have taken very small number of patients for NBUVB therapy alone (only 15). All these studies by Collin et al., Clayton et al. and Mok et al. were retrospective in nature and did not include any prospective post-treatment follow-up. ${ }^{4,5,7}$ Although, Darné et al.'s study was a prospective study, it included only 6 months of post-treatment follow-up. ${ }^{6}$ However, there are no prospective studies with 2 years of post-treatment follow-up for knowing the length of remission and long-term side effects associated with NBUVB in childhood AD. Therefore, we undertook a trial to study the efficacy and safety of NBUVB in childhood AD throughout the therapy and during the post-treatment follow-up period, in terms of reduction in disease intensity, improvement in patient's grade of itch and sleep loss.

\section{METHODS}

\section{Study Design}

The study was a prospective, open labelled clinical trial performed at the Department of Dermatology. The study was approved by the Post-Graduate Board of Medicine and Allied Sciences, Pt. B.D. Sharma Post Graduate Institute of Medical sciences.

\section{Patient selection}

Paediatric patients aged 4 to 14 years having atopic dermatitis diagnosed on basis of the UK refinement of Hanifin and Rajka's diagnostic criteria and having moderate to severe atopic dermatitis (Scoring Atopic Dermatitis; SCORAD index of more than 25) were included in the study. ${ }^{8,9}$ Verbal and written informed consent was taken from patients, parents or guardians.

Exclusion criteria include patients with history of having impaired renal or hepatic function, uncontrolled infected eczema, claustrophobia, intolerance or previous failure to phototherapy, photosentivity or photo-mediated disorders. Patients who had taken other forms of treatment for AD like psoralen therapy, NBUVB, systemic steroids and other immunosuppressant within 3 months were excluded.

A detailed history was taken and dermatological examination was performed to measure the disease intensity and extent SCORAD index was then calculated to assess the severity of disease. ${ }^{9}$ Presence of any other dermatological condition was also noted.

Treatment schedule

Patients were treated with phototherapy in a whole body NBUVB unit (V care UV therapy unit, Bangalore, India) of wavelengths from 310 to $315 \mathrm{~nm}$, containing 16 fluorescent tubes (TL-01) with 100 watts and $6 \mathrm{ft}$. The initial dose of UV-B was $50 \mathrm{~mJ} / \mathrm{cm} 2$, which was determined on the basis of patient's skin type, according to the classification by Pathak et al. ${ }^{10}$ The irradiation dose was increased by $10 \%$ of the previous dose on each subsequent visit. The dose was not increased if there was any trace of erythema. In case of symptomatic erythema with or without oedema/blistering, further treatment was withheld till symptoms subsided. After resolution of symptoms, the dose was reduced to $50 \%$ of the last dose and subsequent increments were by $10 \%$.

During the entire study, no other topical therapy was used other than coconut oil because it does not interfere significantly with NBUVB transmission. ${ }^{11}$ During each treatment, eyes were pro- tected by UV blocking goggles, genitalia and other uninvolved area were shielded.

\section{Clinical assessment}

All the patients were examined by the same dermatologist at each visit. We used a validated scoring system SCORAD index ${ }^{9}$ to accurately monitor $\mathrm{AD}$ disease activity and response to treatment. SCORAD index was calculated at baseline i.e. before starting the therapy and then after $6^{\text {th }}, 12^{\text {th }}, 18^{\text {th }}$ and $24^{\text {th }}$ treatment session. Patients were also followed-up for 2 years and the SCORAD index was calculated at 6 months, 1 year and 2 years of post-treatment follow-up period. During this follow-up period patients did not undergo any phototherapy session and did not receive any other systemic therapy.

We also determined the mean cumulative dose of NBUVB and number of treatment sessions required for clearance. "Clearan$\mathrm{ce}^{\prime \prime}$ was defined when more than $90 \%$ of body surface area was free of the disease..$^{12}$ In our study, the secondary outcomes were measured as improvement in pruritus and reduction in sleep loss. The rating was obtained by using visual analogue score (VAS) consisting of $10 \mathrm{~cm}$ line on which patient indicated a point between "worst itch imaginable/no sleep" and "no itch/ normal sleep" ${ }^{13}$ Adverse effects were looked for at each visit.

\section{Statistical analysis}

All statistical analysis was carried out with Statistical Package for Social Sciences (SPSS) for Microsoft windows $20^{\text {th }}$ version. Descriptive statistics were analysed on the parameters of range, mean \pm standard deviation, frequencies (number of cases), ratio or percentages, whichever was appropriate. The change in mean SCORAD index as compared to baseline at each treatment session and at 6 months, 1 and 2 years of follow-up period was analysed using One-way ANOVA. The improvement in pruritus and reduction in sleep loss from baseline to end of therapy and during the follow-up period was compared using Wilcoxon Signed Ranks test.

\section{Significance of results}

All tests were performed at a $5 \%$ level of significance; thus, an association was significant (S) if the $p$ value was less than 0.05 and $p$ value $<0.001$ as highly significant. The $p$-value more than 0.05 considered as non-significant (NS).

\section{RESULTS}

\section{Patient's Demographic}

Demographics of all the patients are given in table 1. Personal history of atopy was present in $24 \%$ of patients while family history of atopy was present in $16 \%$ of patients. Out of 30 patients, $27(90 \%)$ patients had moderate AD (SCORAD index ranging from $25-50^{9}$ ) while $3(10 \%)$ patients had severe AD (SCORAD index $>50^{9}$ ).

\section{Efficacy evaluation}

All the patients showed improvement in terms of extent of disease, intensity of disease, pruritus and sleep loss. In our study, we observed that SCORAD index had considerably reduced at the $6^{\text {th }}$, $12^{\text {th }}$ and $18^{\text {th }}$ treatment session in comparison to baseline (Table 2). 
TABLE 1: The demographic data of the patients included in the study

Patients $(\mathbf{n}=30)$

Age (years) $8.13+3.22(4-14)$

Mean + SD; Range

Sex ratio (male: female)

Duration of disease (years) Mean + SD; Range $4.23+3.02(1-12)$

Age at onset (years) Mean + SD; Range

$3.90+3.44(0.1-12)$

Body surface area (percentage) Mean $+\mathrm{SD}$;

$34.13+16.90(12-77)$

Range

\begin{tabular}{cccc}
\multicolumn{4}{|c}{ TABLE 2: SCORAD index at baseline, during and after the } \\
therapy & & \\
\hline SCORAD index & Range & Mean + SD & p-value \\
\hline Baseline & $20-56$ & $30.42+7.90$ & - \\
6th treatment session & $7.2-30$ & $14.01+5.80$ & $<0.001$ \\
12th treatment session & $0-22.5$ & $11.41+5.89$ & $<0.001$ \\
18th treatment session & $0-19.1$ & $8.89+4.25$ & $<0.001$ \\
24th treatment session & $0-18.0$ & $7.82+3.72$ & $<0.001$ \\
After 6 month of post-treatment & $0-19$ & $8.09+3.41$ & $<0.001$ \\
After 1 year of post-treatment & $0-20$ & $10.29+3.85$ & $<0.001$ \\
After 2 years of post-treatment & $0-22.4$ & $15.34+5.08$ & $<0.001$ \\
\hline
\end{tabular}

baseline (Table 1). Similarly, during the $1^{\text {st }}$ and $2^{\text {nd }}$ year of post-treatment follow-up period the improvement in pruritus and reduction in sleep loss was maintained both clinically and statistically ( $p$ value $<0.001)$. Although from the baseline the difference of reduction in SCORAD and VAS for pruritus and sleep loss at 6 months was higher in comparison to $1^{\text {st }}$ and $2^{\text {nd }}$ year of post-treatment follow-up, the statistical significance was maintained throughout the whole 2 years of follow-up period (Figure 1, 4 and 5).

\section{Safety evaluation}

Side effects were reported in 5 patients (16.67\%). Grade II erythema was seen in 3 patients. Reactivation of herpes labialis and chickenpox were reported in one patient each. All these patients required temporary cessation of therapy and were restarted on NBUVB at lower doses. During the follow-up period of 2 years the patients were also examined for any side-effects related to NBUVB phototherapy and we did not find any significant adverse effects in this follow-up period.

\section{DISCUSSION}

Children with moderate to severe AD often fail to respond to topical therapy such as emollients, corticosteroids and tacroliyear and 2 years after completion of treatment as compared to the

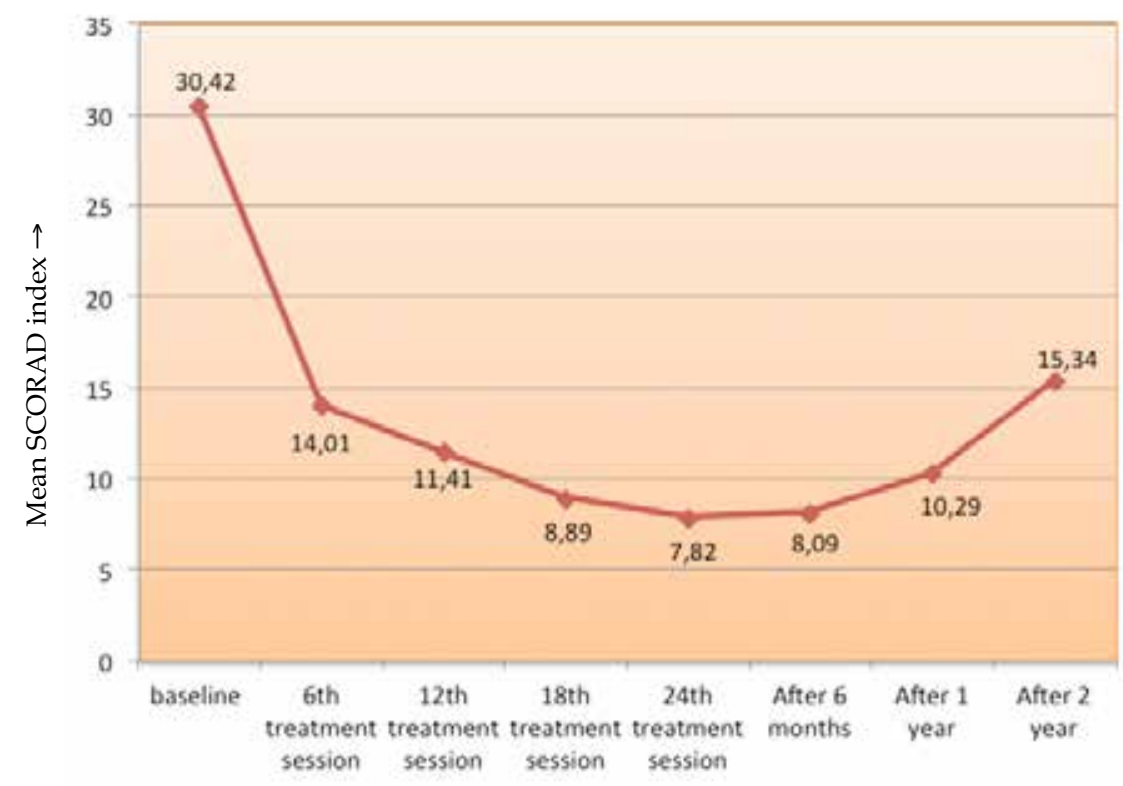

Duration of therapy $\rightarrow$
FiguRE 1: Improvement in SCORAD index with therapy and during post-treatment follow-up period 

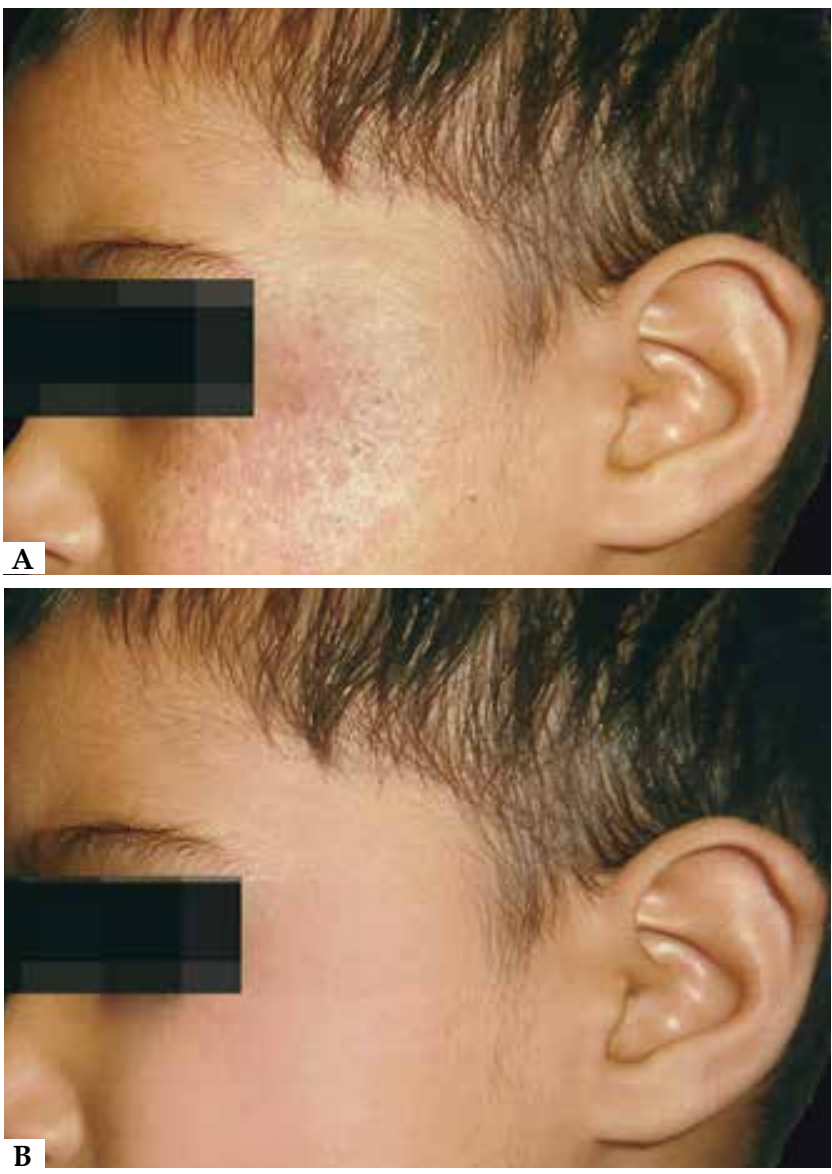

FigURE 2: A. Before NBUVB: showing atopic dermatitis lesion on the left side of the face in a 3 year old male; B. After NBUVB: showing clearance of lesion on the left side of the face

mus. Thus, phototherapy has been regarded as another alternative for the treatment of moderate to severe AD. PUVA with oral 8-Methoxysolaren has been shown to be an effective treatment option for adult $\mathrm{AD}$, although there are concerns regarding its long-term safety. ${ }^{14}$ According to a recent review by Dogra et al. ${ }^{15}$ both UVA1 and NBUVB are equally effective in the treatment of patients with moderate to severe atopic dermatitis. However, UVA1 is preferable for controlling acute flare of atopic dermatitis while NBUVB is most effective in managing chronic disease. Another systematic review by Pérez-Ferriols et al. ${ }^{16}$, described two studies according to which NBUVB was found to be superior than medium dose UV-A1, with a better reduction of pruritus compared to medium dose UVA1. Moreover, UVA1 has potential association with photo-carcinogenesis like melanoma and Merkel cell carcinoma. ${ }^{15}$

NBUVB has been reported to be effective in the management of $\mathrm{AD}$ in adults resulting in prolonged remission. ${ }^{3} \mathrm{In} \mathrm{AD}$, there is an inappropriate balance between $\mathrm{CD}_{4}{ }^{+} \mathrm{CD}_{25}$ high Tregs and $\mathrm{Th}$ Teffs. This imbalance may result from a deficiency in suppression by Tregs or by strong activation signals that supersede the regulatory mechanism. ${ }^{17}$ Samahy et al. ${ }^{18}$ has recently reported a significant reduction of clinical severity grades according to the Leicester score after NB-UVB therapy, with overall significant elevation of Tregs
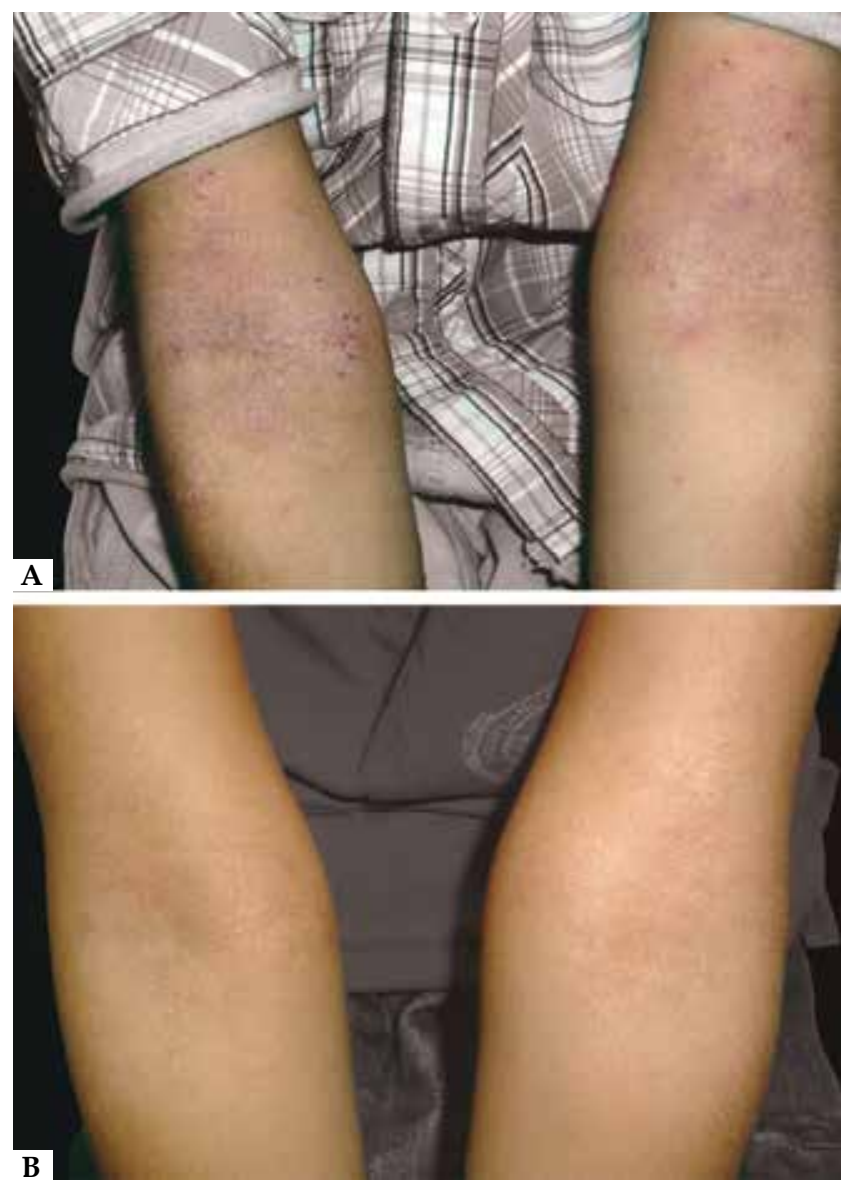

FigURE 3: A. Before NBUVB: lesions of atopic dermatitis on the cubital fossae bilaterally in a 6 year old male; B. After NBUVB: showing clearance of lesions on the cubital fossae

FoxP $\mathrm{P}_{3}$ expression\%. Comparing pre- and post-phototherapy data, there was a statistically significant elevation of Tregs $\%$ and Tregs/ Teffs ratio in mild grade patients and significant elevation of Tregs Fox $\mathrm{P}_{3}$ expression $\%$ and Tregs/Teffs ratio in the moderate group. However, no significant change was detected in the severe group which could be attributed due to the small sample size as well as the high pretreatment Tregs $\%$ in the patients of severe group.

Clinical studies have shown NBUVB phototherapy as an effective and well-tolerated treatment in childhood AD; however, its long-term adverse effects and length of remission have not been thoroughly assessed. ${ }^{4-7,12}$ Furthermore, on perusal of literature, there are very few studies observing the effect of NBUVB in childhood $\mathrm{AD}$ of which most of the studies are retrospective and have included small number of patients. ${ }^{4,5,7,12}$ The present study was, therefore, undertaken to determine the response and safety of NBUVB in children with $\mathrm{AD}$, in terms of efficacy, reduction in disease intensity and extent of disease, adverse effects, amelioration in pruritus and sleep loss.

In our study, out of 30 patients, $90 \%$ had moderate $\mathrm{AD}$ and $10 \%$ had severe AD. This is in concordance with the earlier Indian studies which have also highlighted that severity of AD in Indian patients is much less as compared to that in the West. ${ }^{19,20}$ 

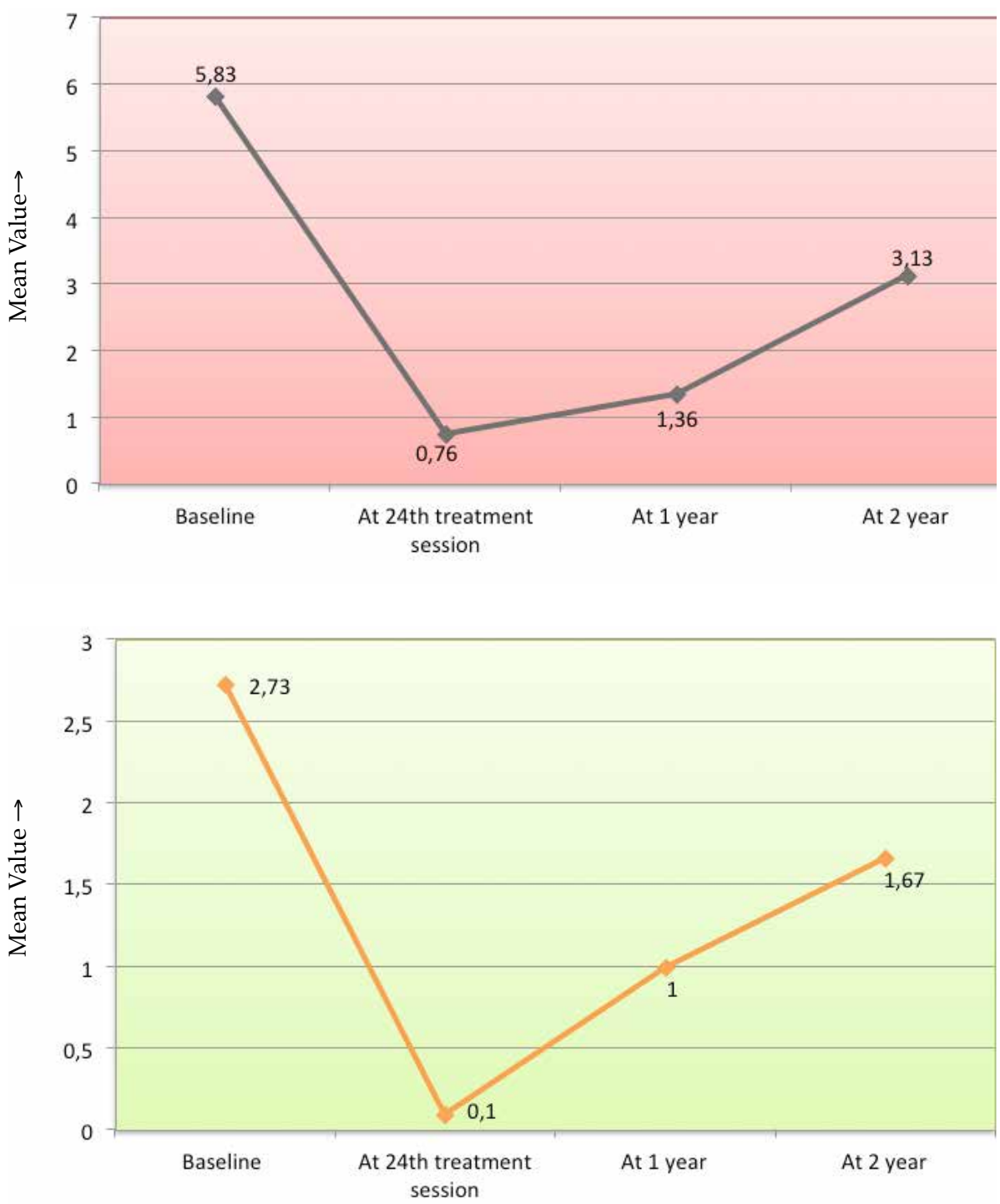

FIgURE 4: Improvement in pruritus measured on VAS during and after the treatment
FIGURE 5: Reduction in sleep loss measured on VAS during and after the treatment
In the present study, we observed that SCORAD index had considerably reduced at the $6^{\text {th }}, 12^{\text {th }}, 18^{\text {th }}$ and $24^{\text {th }}$ treatment session in comparison to baseline. This was in concordance to a recent study by Darné et al. ${ }^{6}$ who had also showed a significant decrease in SCORAD index at 12 weeks of treatment (i.e. end of treatment) as compared to baseline. Besides the study by Darné et al., all other previous studies were retrospective studies and have not used such validated scoring system to measure the improvement in response to NBUVB. ${ }^{4,5,7}$

The results of our study reveal that clearance of disease was seen in $90 \%$ of patients. Maximum number of patients achieved clearance at the $18^{\text {th }}$ treatment session, while Darné et al. had observed that only $41 \%$ of children with AD achieved clearance or minimal residual disease at the end of therapy, i.e., 12 weeks. In our study, all the patients were of Indian origin while in Darné et al. study there was predominance of patients of western origin. Thus, the higher rates of clearance in our study can be explained by the fact that patients of western origin have been reported to have more treatment resistant eczema. ${ }^{6}$

In our study, the maximum cumulative dose of NBUVB for clearance was $2.267 \mathrm{~J} / \mathrm{cm}^{2}$. The cumulative dose in our study is relatively less as compared to other studies because we have given twice weekly NBUVB starting with $50 \mathrm{~mJ} / \mathrm{cm}^{2}$ and session wise increment of $10 \%$ only while in other studies therapy was either given thrice weekly or doses were given according to MED values with $20 \%$ increments three times in a week. ${ }^{5,6,12}$

In our study, secondary outcomes were assessed using restoration of sleep and reduction in pruritus. Pruritus showed significant improvement from baseline to the end of three months of therapy and during two years of post-treatment follow-up period. Similarly, significant restoration in normal sleep pattern was seen at the end of therapy, i.e., 3 months, and during the two years post-treatment follow-up period. These results were in agreement 
with Darné et al. study where they also mentioned amelioration of pruritus and restoration of normal sleep pattern by the patients and parents as treatment benefits during and after 6 months of therapy. ${ }^{6}$

In the present study, when we assessed the adverse effects, we found that the therapy was generally well tolerated during and after the treatment. The side effect profile observed in our study was in agreement with the earlier studies. Clayton et al. have reported that NBUVB phototherapy was generally well tolerated except for short term adverse effects in few patients like grade II erythema and reactivation of Herpes simplex virus, etc. ${ }^{5}$ Likewise, Mok et al. had reported minor side effects like erythema, skin peeling and itch. ${ }^{7}$ Darné et al. had also concluded that side effects were mild, mainly in the form of erythema. ${ }^{6}$ But concern remains about the long-term carcinogenesis potential of NBUVB. This made us follow-up these patients for two years after NBUVB, however we did not find any side effects during this period.

Our study holds considerable importance because the study group was made of children, and therefore there is need for a treatment that is effective as well as safe. The use of validated scoring system, i.e., SCORAD in our study has ensured the accurate monitoring of $\mathrm{AD}$ disease activity. Furthermore, to the best of our knowledge, there are no prospective studies available in the literature with such a long term, i.e., 2 years of post-treatment follow-up period. However, there were some limitations in our study. Firstly, the study was an open-label trial, not randomized nor controlled. A control group was not used as we felt it was unethical not to give any treatment to children afflicted with moderate to severe atopic dermatitis. Secondly, the study period was relatively short, in terms of post-treatment follow-up period. Thus, one would require large multicentric studies involving several thousand new patients per year followed-up for 10 years or more, to determine its carcinogenic potential.

\section{CONCLUSIONS}

Our study proves that NBUVB phototherapy is an efficacious and safe modality of treatment in childhood AD. It also reaffirms the school of thought that it could be relied upon as the first line therapy in moderate to severe $\mathrm{AD}$ in the pediatric age group before opting for more toxic therapies as it has a good therapeutic index and low profile of acute and possibly long-term side effects. $\square$

\section{REFERENCES}

1. Nutten S. Atopic dermatitis: global epidemiology and risk factors. Ann Nutr Metab 2015;66:8-16

2. Darsow U, Wollenberg A, Simon D, Taïeb A, Werfel T, Oranje A, et al. ETFAD/ EADV eczema task force 2009 position paper on diagnosis and treatment of atopic dermatitis. J Eur Acad Dermatol Venereol. 2010;24:317-28.

3. Gambichler T, Breuckmann F, Boms S, Altmeyer P, Kreuter A. Narrowband UVB phototherapy in skin conditions beyond psoriasis. J Am Acad Dermatol. 2005:52:660-70.

4. Collins P, Ferguson J. Narrowband (TLO1) UVB air-conditioned phototherapy for atopic eczema in children. Br J Dermatol. 1995;133:653-5.

5. Clayton TH, Clark SM, Turner D, Goulden V. The treatment of severe atopic dermatitis in childhood with narrowband ultraviolet B phototherapy. Clin Exp Dermatol. 2007;32:28-33.

6. Darné S, Leech SN, Taylor AE. Narrowband ultraviolet B phototherapy in children with moderate to severe eczema: a comparative cohort study. Br J Dermatol. 2014;170:150-6.

7. Mok ZR, Koh MJ, Chong WS. Is phototherapy useful in the treatment of atopic dermatitis in asian children? A 5-year report from singapore. Pediatr Dermatol. 2014:31:698-702.

8. Friedmann PS, Ardern-Jones MR, Holden CA. Atopic Dermatitis. In: Burns T, Breathnach S, Cox N, Griffiths C, editors. Rook's Textbook of Dermatology. 8th ed. Oxford: Wiley Blackwell; 2010. p.24.1-8.

9. Oranje AP, Glazenburg EJ, Wolkerstorfer A, de Waard-van der Spek FB. Practical issues on interpretation of scoring atopic dermatitis: the SCORAD index, objective SCORAD and the three item severity score. Br J Dermatol. 2007;157:645-8.

10. Pathak MA, Jimbow K, Szabo G, Fitzpatrick TB. Sunlight and melanin pigmentation. In: Smith KC, editor. Photochemical and photobiological reviews. New York: Plenum; 1976. p.211-39.

11. George SA, Bilsland DJ, Wainwright NJ, Ferguson J. Failure of coconut oil to accelerate psoriasis clearance in narrow-band UVB phototherapy or photochemotherapy. Br J Dermatol. 1993;128:301-5

12. Tay YK, Morelli JG, Weston WL. Experience with UVB phototherapy in children Pediatr Dermatol. 1996;13:406-9.
13. Chrostowska-Plak D, Salomon J, Reich A, Szepietowski JC. Clinical aspects of itch in adult atopic dermatitis patients. Acta Derm Venereol. 2009:89:379-83.

14. Stern RS, Lunder EJ. Risk of squamous cell carcinoma and methoxsalen (psoralen) and UV-A radiation (PUVA): a meta-analysis. Arch Dermatol. 1998;134:1582-5.

15. Dogra S, Mahajan R; Indian Association of Dermatologists, Venereologists and Leprologists. Phototherapy for atopic dermatitis. Indian J Dermatol Venereol Leprol. 2015;81:10-5.

16. Pérez-Ferriols A, Aranegui B, Pujol-Montcusí JA, Martín-Gorgojo A, CamposDomínguez M, Feltes RA, et al. Phototherapy in atopic dermatitis: a systematic review of the literature. Actas Dermosifiliogr. 2015;106:387-401.

17. Ling EM, Smith T, Nguyen XD, Pridgeon C, Dallman M, Arbery J, et al. Relation of CD4+CD25 + regulatory T-cell suppression of allergen driven T-cell activation to atopic status and expression of allergic disease. Lancet. 2004;363:608-15.

18. El Samahy MH, Attia EA, Saad AA, Mahmoud EY. Circulating CD4(+) CD25(high) FoxP3(+) T-regulatory cells in patients with atopic dermatitis after narrowbandultraviolet B phototherapy. Int J Dermatol. 2015;54:e424-9.

19. Kanwar AJ, Dhar S. Severity of atopic dermatitis in India. $\mathrm{Br} \mathrm{J}$ Dermatol. 1994:131:733-4.

20. Dhar S, Banerjee R, Dutta AK, Gupta AB. Comparison between the severity of atopic dermatitis in Indian children born and bought up in UK and USA and that of Indian Children born and bought up in India. Indian J Dermatol. 2003;48:200-2.

\author{
MAILING ADDRESS: \\ Dr.Surabhi Dayal \\ Pt.B.D.Sharma Post Graduate Institute of Medical Sciences, \\ Rohtak-124001, Haryana, India \\ E-mail:surabhidayal7@gmail.com
}

How to cite this article: Dayal S, Pathak K, Sahu P, Jain VK. Narrowband UV-B phototherapy in childhood atopic dermatitis: efficacy, safety and post-treatment follow-up. An Bras Dermatol. 2017;92(6):801-6. 